\title{
Intracranial Leptomeningeal Carcinomatosis from Breast Cancer Detected on ${ }^{18}$ F-FDG PET
}

\author{
Bradley J. Carra and Michael N. Clemenshaw
}

Department of Nuclear Medicine, San Antonio Military Medical Center, Fort Sam Houston, Texas

Leptomeningeal carcinomatosis is an uncommon manifestation of non-central nervous system (CNS) metastatic disease. Diagnosis, however, has important prognostic and treatment implications. We present a case in which intracranial leptomeningeal carcinomatosis from a primary breast cancer was detected with ${ }^{18} \mathrm{~F}-\mathrm{FDG} \mathrm{PET} / \mathrm{CT}$, despite its low sensitivity for detection of CNS metastases from non-CNS primary tumors.

Key Words: ${ }^{18}$ F-FDG PET/CT; leptomeningeal carcinomatosis; breast cancer

J Nucl Med Technol 2015; 43:227-228

DOI: $10.2967 /$ jnmt.114.148791

$\mathbf{L}$

eptomeningeal carcinomatosis (LC) is an uncommon late manifestation of non-central nervous system (CNS) solid malignancies. ${ }^{18} \mathrm{~F}-\mathrm{FDG}$ PET/CT has a low sensitivity for detection of CNS metastases from non-CNS primary tumors (1). ${ }^{18} \mathrm{~F}-\mathrm{FDG}$ PET/CT detection of LC from other non-CNS primary tumors has been described (1), and a few cases of ${ }^{18} \mathrm{~F}-\mathrm{FDG}$ PET/CT detection of spinal LC from breast cancer $(2,3)$ have been reported. To our knowledge, however, this is the first reported case of intracranial LC from breast cancer detected with ${ }^{18} \mathrm{~F}-\mathrm{FDG}$ PET/CT.

\section{CASE REPORT}

A 38-y-old woman with stage IV inflammatory ductal carcinoma of the breast metastatic to the bones presented with ataxia 2 wk after completion of palliative chemotherapy. Contrast-enhanced brain MR imaging demonstrated nodular leptomeningeal enhancement primarily of the cerebellar folia (Fig. 1). A differential diagnosis of LC versus infectious meningitis was provided. A total of 4 cerebrospinal fluid (CSF) samples were negative for infection and metastatic disease.

${ }^{18} \mathrm{~F}-\mathrm{FDG}$ PET/CT was performed for restaging purposes. Imaging from the skull vertex through the mid femora was

\footnotetext{
Received Sep. 19, 2014; revision accepted Nov. 13, 2014.

For correspondence or reprints contact: Bradley J. Carra, San Antonio Military Medical Center, 3551 Roger Brooke Dr., Fort Sam Houston, TX 78234.

E-mail: bradley.j.carra.mil@mail.mil

Published online Feb. 5, 2015.

COPYRIGHT (c) 2015 by the Society of Nuclear Medicine and Molecular Imaging, Inc.
}

begun 90 min after intravenous injection of $384 \mathrm{MBq}$ of ${ }^{18} \mathrm{~F}-\mathrm{FDG}$. Imaging revealed increased ${ }^{18} \mathrm{~F}-\mathrm{FDG}$ avidity conforming to the sites of leptomeningeal enhancement on brain MR imaging, as well as progression of osseous metastatic disease (Fig. 2). Despite negative CSF cytology results, the presence of other metastatic disease detected on ${ }^{18} \mathrm{~F}$-FDG PET/CT and a lack of infectious symptoms supported a diagnosis of LC. Neurologic symptoms rapidly regressed after placement of a ventriculostomy and initiation of high-dose steroids and intrathecal chemotherapy.

\section{DISCUSSION}

The most common intracranial locations of CSF tumor seeding are the basilar cisterns and cerebellum. Although breast cancer is the most common nonhematologic tumor

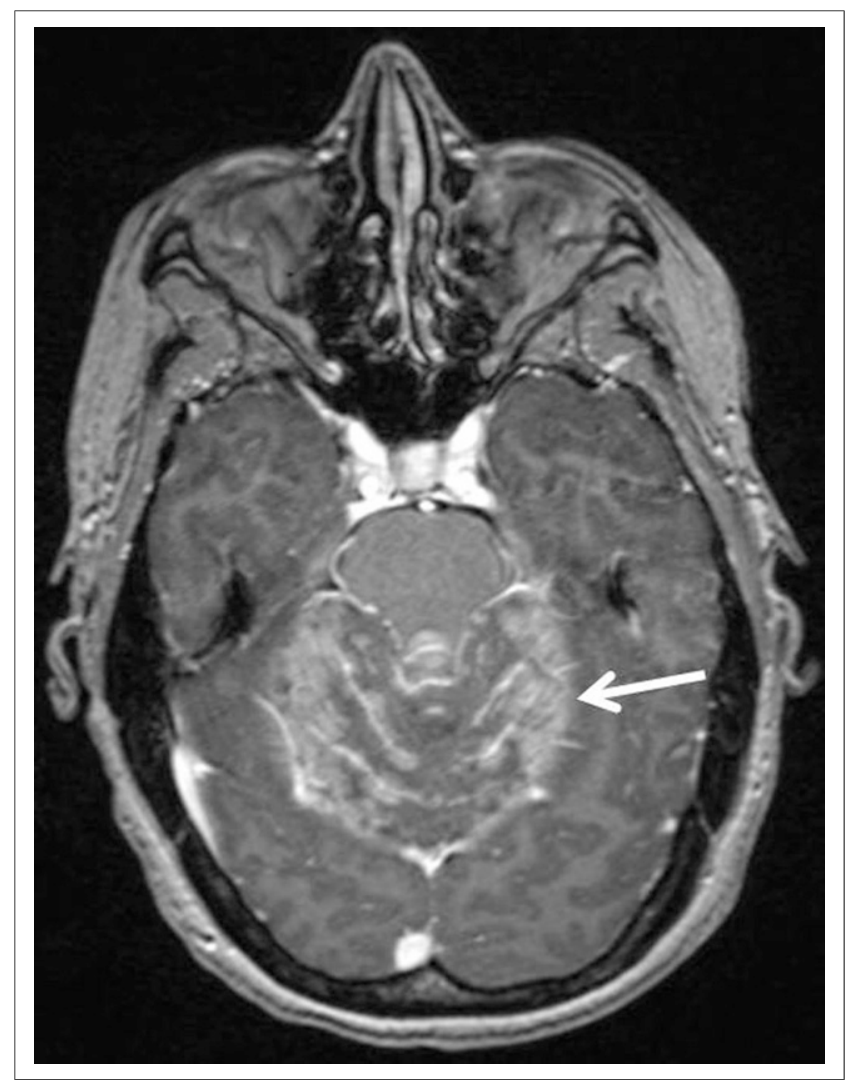

FIGURE 1. Postcontrast axial T1-weighted image of brain demonstrating leptomeningeal enhancement of cerebellar folia (arrow). 


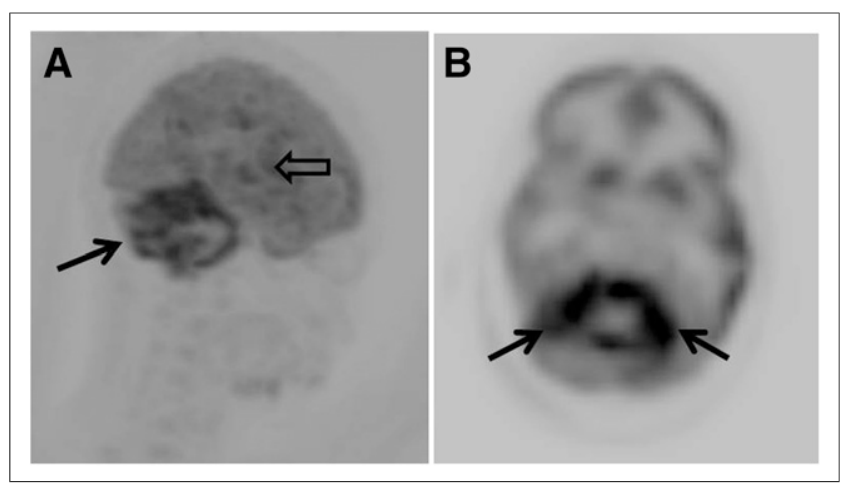

FIGURE 2. Maximum intensity projection (A) and axial image (B) from ${ }^{18} \mathrm{~F}-\mathrm{FDG}$ PET showing increased leptomeningeal radiotracer uptake in cerebellum (solid arrows) and temporal lobe (open arrow).

with leptomeningeal spread, the incidence remains low at only $1 \%-5 \%(4,5)$. LC has a poor prognosis, with median survival of only $2-4$ mo (2).

Detection of metastatic cells within the CSF remains the gold standard for diagnosis, but the yield is poor, with detection rates of only $50 \%$ on initial CSF sampling (4). Contrast-enhanced MR imaging is the imaging modality of choice for diagnosis showing leptomeningeal enhancement. Despite its low sensitivity, ${ }^{18} \mathrm{~F}-\mathrm{FDG}$ PET/CT may show increased leptomeningeal metabolic activity (1).

Leptomeningeal enhancement and increased ${ }^{18} \mathrm{~F}-\mathrm{FDG}$ avidity are both nonspecific findings and can be seen in infectious meningitis, LC, or neurosarcoidosis. In conjunction with clinical information, however, they can suggest the diagnosis of LC. Although MR imaging is likely more sensitive in detecting LC, whole-body ${ }^{18}$ F-FDG PET/CT has the advantage of demonstrating other metastatic disease, an important finding because LC is usually a late manifestation of metastatic disease and less commonly seen in isolation (5).

\section{CONCLUSION}

Although the sensitivity of ${ }^{18}$ F-FDG PET/CT for detection of CNS metastases is poor, routine staging examinations cover the most common locations of intracranial leptomeningeal metastases. Detection is therefore possible and has important prognostic and treatment implications. Although MR imaging is the imaging modality of choice for diagnosing leptomeningeal metastasis, whole-body ${ }^{18} \mathrm{~F}-\mathrm{FDG}$ PET/CT is uniquely able to detect other systemic metastatic disease, important information supporting a diagnosis of LC.

\section{DISCLOSURE}

No potential conflict of interest relevant to this article was reported.

\section{REFERENCES}

1. Rohren EM, Provenzale JM, Barboriak DP, Coleman RE. Screening for cerebral metastases with FDG PET in patients undergoing whole-body staging of non-central nervous system malignancy. Radiology. 2003;226: $181-187$.

2. Shah S, Rangarajan V, Purandare N, Luthra K, Medhi S. ${ }^{18}$ F-FDG uptakes in leptomeningeal metastases from carcinoma of the breast on a PET/CT study. Indian J Cancer. 2007;44:115-118.

3. Grande ML, Rayo J, Serrano J, Infante J, Garcia L, Duran C. Leptomeningeal carcinomatosis as only pathological finding at FDG-PET/CT in case of tumor marker elevation in breast cancer. Indian J Nucl Med. 2014;29:53-54.

4. Wasserstrom WR, Glass JP, Posner JB. Diagnosis and treatment of leptomeningeal metastases from solid tumors. Cancer. 1982;49:759-772.

5. Boogerd W, Hart AAM, vanderSande JJ, Engelsman E. Meningeal carcinomatosis in breast cancer. Cancer. 1991;67:1685-1695. 